\title{
Status of eV-scale sterile neutrinos
}

\section{Yu-Feng Li ${ }^{*}$}

Institute of High Energy Physics, and School of Physical Sciences, University of Chinese Academy of Sciences, Beijing 100049, China

E-mail: liyufengeihep.ac.cn

The LSND, Gallium radioactive source results as well as the reactor rates and spectral ratios data can be explained by short-baseline neutrino oscillations due to the mixing of the active neutrinos with sterile neutrinos at the $\mathrm{eV}$ scale. I briefly review the theory of neutrino oscillations in the framework of $(3+1)$ mixing with one sterile neutrino and the latest status of a $(3+1)$ global fit of short-baseline neutrino oscillation data.

Neutrino Oscillation Workshop (NOW2018)

9 - 16 September, 2018

Rosa Marina (Ostuni, Brindisi, Italy)

${ }^{*}$ Speaker. 


\section{Introduction}

The LSND [1], Gallium radioactive source [2] anomalous experimental results as well as the reactor rate [3] and spectral ratio [4] anomalies are intriguing indications in favor of short-baseline (SBL) neutrino oscillations due to sterile neutrinos at the $\mathrm{eV}$ scale. Here I consider the simplest $(3+1)$ active-sterile neutrino mixing [5], in which there are three massive neutrinos $v_{i}(i=1,2,3)$ which are the main constituents of the three standard active neutrinos, and there is a fourth massive neutrino $v_{4}$ at the $\mathrm{eV}$ scale which is mainly a sterile flavor state $\left(v_{4} \simeq v_{s}\right)$.

Since the neutrino squared-mass difference responsible for the SBL oscillations at the scale of $1 \mathrm{eV}^{2}$ is much larger than those of solar and atmospheric squared-mass differences which are located at the scales of $7.5 \times 10^{-5} \mathrm{eV}^{2}$ and $2.5 \times 10^{-3} \mathrm{eV}^{2}$ respectively, the (3+1) active-sterile mixing scheme is a perturbation of the standard three-neutrino mixing with $\left|U_{\alpha 4}\right|^{2} \ll 1(\alpha=e, \mu, \tau)$ and $\Delta m_{41}^{2} \simeq \Delta m_{42}^{2} \simeq \Delta m_{43}^{2}$ with $\Delta m_{j k}^{2} \equiv m_{j}^{2}-m_{k}^{2}$. Therefore, the effective oscillation probabilities of the flavor neutrinos can be written as [5]

$$
P_{\bar{v}_{\alpha} \rightarrow \bar{v}_{\beta}}^{(\mathrm{SBL})} \simeq\left|\delta_{\alpha \beta}-\sin ^{2} 2 \vartheta_{\alpha \beta} \sin ^{2} \frac{\Delta m_{41}^{2} L}{4 E}\right|,
$$

where $\alpha, \beta=e, \mu, \tau, L$ is the source-detector distance and $E$ is the neutrino energy. The SBL neutrino oscillation amplitudes are defined as

$$
\sin ^{2} 2 \vartheta_{\alpha \beta}=4\left|U_{\alpha 4}\right|^{2}\left(\delta_{\alpha \beta}-\left|U_{\alpha 4}\right|^{2}\right) .
$$

Here I review the updated results of the $(3+1)$ global fit of SBL neutrino oscillation data presented in Ref. [6], which include the latest experimental results from MiniBooNE, MINOS/MINOS+ and other SBL reactor antineutrino data.

In the global fit, we consider the following three groups of experiments:

(A) The muon-to-electron neutrino and antineutrino appearance data of the LSND, MiniBooNE, BNL-E776, KARMEN, NOMAD, ICARUS and OPERA experiments.

(B) The following electron neutrino and antineutrino disappearance data: 1) The ratios of measured and predicted rates of the short-baseline reactor antineutrino experiments; 2) The electron antineutrino energy spectra measured in the Bugey-3 and NEOS and DANSS short-baseline reactor experiments; 3) the data of GALLEX and SAGE Gallium radioactive source experiments; 4) the solar neutrino constraint on $\sin ^{2} 2 \vartheta_{e e}$; 5) the KARMEN and LSND $v_{e}$-C charged current data.

(C) The muon neutrino and antineutrino disappearance data obtained from the CDHSW experiment, from the data of atmospheric neutrino oscillation experiments, from the analysis of the SciBooNE-MiniBooNE neutrino and antineutrino data, and the latest constraints from the MINOS/MINOS+ and IceCube experiments.

\section{Electron neutrino and antineutrino disappearance}

The Gallium radioactive source data in the GALLEX and SAGE solar neutrino experiments have shown a $2.9 \sigma$ deficit for the measured neutrino events compared to the predictions, where the averaged rate turns out to be $0.84 \pm 0.05$ [2]. Meanwhile, the reactor rate measurements show a consistent deficit of $6 \%$ compared to the prediction of the Huber and Muller model [7], in which 

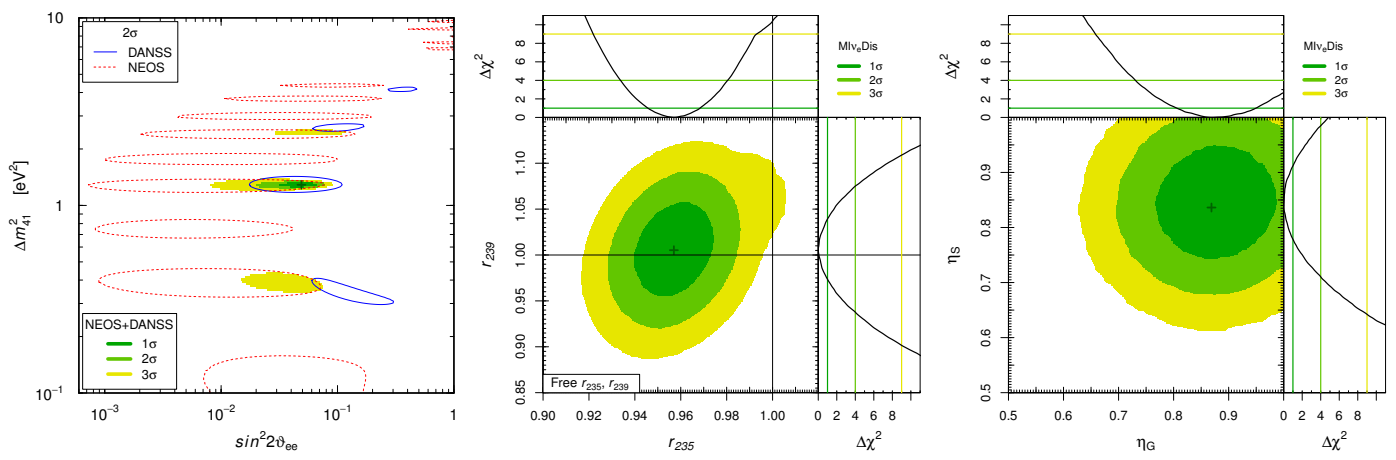

Figure 1: The combined analysis of the NEOS and DANSS spectral ratio data (left panel) and the MI $v_{e}$ Dis fitting results of the the isotopic cross sections per fission (middle panel) and the efficiencies of the GALLEX and SAGE detectors (right panel).

the significance would be $2.8 \sigma$ [3] if the nominal systematic uncertainties are assumed for the aforementioned model. However, the new measurement reactor spectral bump [8] and fuel fraction information [9] have raised critical questions on the validity of the model predictions.

More recently, the reactor spectral ratio data in NEOS and DANSS have shown interesting indications of the SBL oscillations with the significance of around $3 \sigma$, where in the left panel of Fig. 1, a combined analysis of the NEOS and DANSS spectral ratio data is presented with the best fit point located at $\Delta m_{41}^{2} \simeq 1.3 \mathrm{eV}^{2}$ and $\sin ^{2} 2 \vartheta_{e e} \simeq 0.05$ [4]. Since this oscillation amplitude only contributes to around $2.5 \%$ of the total deficit in the electron neutrino and antineutrino disappearance, we need additional contributions to explain the Gallium and reactor anomalies. Thus a model independent $v_{e}$ disappearance ( $\left.\mathrm{MI} v_{e} \mathrm{Dis}\right)$ analysis framework [4] is proposed to include both the oscillation effect and the corrections to the isotopic cross sections per fission $\left(r_{235}\right.$ and $\left.r_{239}\right)$ and efficiencies of the GALLEX and SAGE detectors $\left(\eta_{\mathrm{G}}\right.$ and $\left.\eta_{\mathrm{S}}\right)$. The middle and right panels of Fig. 1 are shown for the fitting results of the the isotopic cross sections per fission and the efficiencies of the GALLEX and SAGE detectors, respectively. Therefore, the hybrid scenarios including both the oscillation and corrections of the neutrino fluxes or detector efficiencies are preferred.

\section{Global fits}

The LSND electron antineutrino appearance signal [1] favors a SBL oscillation explanation with $\Delta m_{41}^{2} \simeq 1 \mathrm{eV}^{2}$ and $\sin ^{2} 2 \vartheta_{e \mu} \simeq 0.002$. The MiniBooNE experiment [10] was designed to test the LSND signal with rather different neutrino energies and baselines, but similar $L / E$ ratios. However, both the neutrino and antineutrino data of MiniBooNE have been inconclusive, which did not confirm the LSND signal in the similar $L / E$ range, but observed an anomalous event excess in the low energy range. This low energy excess can not be explained by the SBL oscillations due to the strong limits from disappearance channels. The future MicroBooNE experiment is designed to test whether the low energy excess events are photon-like or electron-like signals.

In the muon neutrino and antineutrino disappearance channel, the latest strong negative limits from IceCube [11] and MINOS/MINOS+ [12] experiments have been published for the SBL oscillation searches, which have increased the appearance and disappearance tension to an intolerable level. The global status of the $(3+1)$ active sterile oscillation model is shown in Fig. 2, where the 


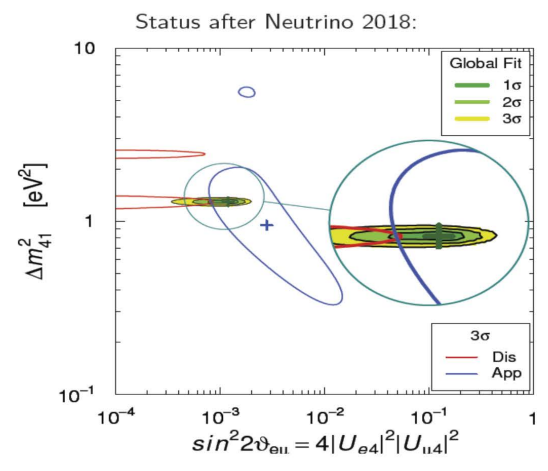

Figure 2: Global status of the $(3+1)$ active sterile oscillation model. The strong tension between the appearance and disappearance channels are illustrated in the enlarged circle.

strong tension between the appearance and disappearance channels are illustrated in the enlarged circle.

\section{Conclusion}

The reactor spectral ratio data in NEOS and DANSS have shown interesting model independent indications of short baseline oscillations, and if it will be confirmed will demand a hybrid solution to the reactor flux and Gallium anomalies. On the other hand, the increasing negative limits of muon neutrino disappearance measurements are in strong tension with the oscillation-based explanations of LSND and MiniBooNE appearance signals. Finally Let me emphasize that the confirmation of the existence of sterile neutrinos would be a major discovery which would have a profound impact not only on the neutrino physics, but on the whole view of fundamental physics.

\section{References}

[1] A. Aguilar-Arevalo et al. [LSND Collaboration], Phys. Rev. D 64, 112007 (2001).

[2] C. Giunti and M. Laveder, Phys. Rev. C 83, 065504 (2011).

[3] G. Mention et al., Phys. Rev. D 83, 073006 (2011).

[4] S. Gariazzo, C. Giunti, M. Laveder and Y. F. Li, Phys. Lett. B 782, 13 (2018).

[5] S. Gariazzo, C. Giunti, M. Laveder, Y. F. Li and E. M. Zavanin, J. Phys. G 43, 033001 (2016).

[6] S. Gariazzo, C. Giunti, M. Laveder and Y. F. Li, JHEP 1706, 135 (2017).

[7] T. A. Mueller et al., Phys. Rev. C 83, 054615 (2011); P. Huber, Phys. Rev. C 84, 024617 (2011).

[8] F. P. An et al. [Daya Bay Collaboration], Phys. Rev. Lett. 116, no. 6, 061801 (2016).

[9] F. P. An et al. [Daya Bay Collaboration], Phys. Rev. Lett. 118, no. 25, 251801 (2017).

[10] A. A. Aguilar-Arevalo et al. [MiniBooNE Collaboration], Phys. Rev. Lett. 121, no. 22, 221801 (2018).

[11] M. G. Aartsen et al. [IceCube Collaboration], Phys. Rev. Lett. 117, no. 7, 071801 (2016).

[12] P. Adamson et al. [MINOS Collaboration], [arXiv:1710.06488 [hep-ex]]. 